\title{
STUDY OF STRUCTURE AND ELECTRICAL PROPERTIES OF $\mathrm{Bi}_{2} \mathrm{Te}_{3}$
}

\section{R. R. Urkude and U. A. Palikundwar}

Department of Physics, Rashtrasant Tukadoji Maharaj Nagpur University, Nagpur33. rajashriurkude@yahoo.co.in, uapali@yahoo.com

\section{Abstract}

$\mathrm{Bi}_{2} \mathrm{Te}_{3}$ alloys are the best thermoelectric materials having important technological applications near the room temperature. Bulk samples of $\mathrm{Bi}_{2} \mathrm{Te}_{3}$ were synthesized by a method, similar to the solvothermal method, at different reaction temperatures and reaction time intervals. The reaction between $\mathrm{BiCl}_{3}, \mathrm{Te}$, and $\mathrm{NaBH}_{4}$ in $\mathrm{N}, \mathrm{N}$-dimethylformamide at 373-453 $\mathrm{K}$ was used to form the alloys; $\mathrm{NaBH}_{4}$ was used as a reducing agent. The final product was filtered, washed, dried and pressed to compacted pellets and was followed by a sintering at $500 \mathrm{~K}$. The samples were characterized by X-ray diffraction method and electrical conductivity measurements were carried out.

Keywords: Bismuth telluride; thermoelectric materials, Electrical conductivity.

\section{Introduction}

The bismuth telluride alloys are the best known thermoelectric materials for applications near room temperature [1]. They have many important applications e.g. used as thermopiles and thermal sensors, thermoelectric cooler for laser diodes [2-5]. The actual efficiency of these devices are too low, however, theoretical [6, 7] and experimental results [8] have shown that the quantum confinement effects in nanostructured materials can improve the efficiency of thermoelectric materials. Therefore, the synthesis of nanostructural $\mathrm{Bi}_{2} \mathrm{Te}_{3}$ and its alloys has been the focus of recent research.

A few methods have been developed to prepare bismuth telluride, like hydrothermal method [9], self flux technique [10], electrodeposition method [11], solvothermal method [12] to form single crystals, nanocrystalline, thin films etc.

The solvothermal method is a promising approach to obtain many kinds of nanocrystalline non-oxide materials under comparatively low temperature. This method does not need organometallic precursors. In this paper, we have developed a simple method, similar to a solvothermal 
method to synthesize bulk $\mathrm{Bi}_{2} \mathrm{Te}_{3}$ with different mild conditions. The method will be further optimized to synthesize nanostructured $\mathrm{Bi}_{2} \mathrm{Te}_{3}$ alloys.

\section{Review}

Bismuth telluride $\left(\mathrm{Bi}_{2} \mathrm{Te}_{3}\right)$ and its alloys are the best bulk thermoelectric materials. It is a unique material with a potential for wide range of applications. Since the discovery of its extraordinary thermoelectric properties, $\mathrm{Bi}_{2} \mathrm{Te}_{3}$ has played a vital role in thermoelectric industries [13-15].

Bismuth telluride is an anisotropic crystal of hexagonal close-packed $(3 \mathrm{~m})$ structure. Because of this anisotropy the simplest models of the constant-energy surfaces will not explain its transport properties. The electrical and thermal conductivity of the materials is generally highly anisotropic. The model used for the energy-band structure of $\mathrm{Bi}_{2} \mathrm{Te}_{3}$ was proposed by Drabble and Wolfe [16] and used successfully by them to interpret magnetoresistance data in $\mathrm{Bi}_{2} \mathrm{Te}_{3}$. The materials in which this effect has been observed are all crystals of hexagonal (or rhombohedral) symmetry.

Goldsmid [17] observed that the thermoelectric power was anisotropic which depend on the range of temperature and the level of doping. He has performed an experiment on iodine-doped n-type $\mathrm{Bi}_{2} \mathrm{Te}_{3}$, however, thermoelectric power was isotropic in undoped p-type material.

Surface states in $\mathrm{Bi}_{2} \mathrm{Te}_{3}$ exhibit large deviations from a simple electronic band structure due to a combination of smaller band gap $(0.15$ eV) and a strong trigonal potential, which has been utilized to explore some aspects of its surface properties [18-20]. The hexagonal deformation of the surface states was confirmed by STM measurements [21]. An application within this class of materials, $\mathrm{Bi}_{2} \mathrm{Te}_{3}$, is already well known to materials scientists working on thermoelectricity. It is a commonly used thermoelectric material in the engineering near room temperature.

Groshens and co-workers [22] reported a different approach to the synthesis of $\mathrm{Bi}_{2} \mathrm{Te}_{3}$ by elimination reactions conducted in hexane at $503 \mathrm{~K}$. Using organometallic precursors is another route to synthesized $\mathrm{Bi}_{2} \mathrm{Te}_{3}$ [23]. Qian and co-workers [24] reported a solvothermal reaction of metal oxalates 
$\mathrm{Bi}_{2}\left(\mathrm{C}_{2} \mathrm{O}_{4}\right)_{3}$ with $\mathrm{Te}$ in organic solvents are relatively low temperature (393$433 \mathrm{~K})$ to produce crystalline $\mathrm{Bi}_{2} \mathrm{Te}_{3}$.

Few years ago an interest to the stacked quasi-2D layers of bismuth telluride received an additional impetus from a totally different direction. It has been shown that stacks of quasi-2D layers of Te-Bi-Te-Bi-Te are members of a new type of recently discovered materials referred to as topological insulators [25, 26]. The surface state of a quasi-2D crystal of $\mathrm{Bi}_{2} \mathrm{Te}_{3}$ is predicted to consist of a single Dirac cone. Moreover, it has been shown that the layered structures of related materials such as $\mathrm{Bi}_{2} \mathrm{Se}_{3}$ and $\mathrm{Sb}_{2} \mathrm{Te}_{3}$ are also topological insulators.

\section{Experimental Methods}

All the ingredients used in the work were of AR grade and used without further purifications. A mixture of $\mathrm{BiCl}_{3}(10 \mathrm{mmol})$, tellurium (Te) powder (15 mmol), KOH $(80 \mathrm{mmol})$ and $\mathrm{NaBH}_{4}(30 \mathrm{mmol})$ were put into a beaker of $100 \mathrm{ml}$ capacity. The beaker was then filled with $\mathrm{N}, \mathrm{N}$ dimethylformamide (DMF) up to $90 \mathrm{ml}$ and was kept into a muffle furnace. Temperature of the furnace was maintained at 373-453 $\mathrm{K}$ for $10-50$ hours and then slowly cooled to the room temperature. The products were filtered and washed with double distilled water. Finally, the dark products were dried, pressed and sintered at 500 K. X-ray diffraction (XRD) characterization of the samples was performed by recording Hull patterns with help of a Laue camera. Data were collected on a Philips X-ray generator using $\mathrm{Cu} \mathrm{K}$ radiation $\left(\lambda=1.54178 \mathrm{~A}^{\circ}\right)$. The electrical conductivity, in the temperature range of 300-500 K, was measured on compacted pellets of the samples by standard four-probe method with constant current source kept at $2 \mathrm{~mA}$.

\section{Results and discussion}

The Hull pattern of $\mathrm{Bi}_{2} \mathrm{Te}_{3}$ is as shown in Fig. 1 . The rings in the pattern are the Bragg reflections corresponding to the different crystallographic planes. All the planes can be indexed in the space group $\mathrm{R}^{-} \mathrm{m}$ with rhombohedral symmetry. $2 \theta$ values for the reflections with high 
intensity (major peaks) are observed at $21.80^{\circ}, 28.36^{\circ}, 38.65^{\circ}, 42.61^{\circ}$ and the corresponding planes are $\left(\begin{array}{lll}0 & 1 & 2\end{array}\right),\left(\begin{array}{lll}0 & 1 & 5\end{array}\right),\left(\begin{array}{lll}1 & 0 & 10\end{array}\right),\left(\begin{array}{lll}1 & 1 & 0\end{array}\right)$ respectively. The cell parameters calculated from these planes are $a=4.24 \mathrm{~A}^{\circ}$ and $c=$ $30.45 \mathrm{~A}^{\circ}$, which are in good agreement with the reported values $a=4.385$ $\mathrm{A}^{\circ}, c=30.48 \mathrm{~A}^{\circ}$ in JCPDS card No. 15-0863 [12]. It can be confirmed from this study that the $\mathrm{Bi}_{2} \mathrm{Te}_{3}$ samples, as prepared by our method, are single phase with space group $\mathrm{R}^{-} \mathrm{m}$ and rhombohedral symmetry. There is no major difference in the Hull patterns of the samples prepared with different temperatures and reaction time intervals. Table 1 shows the $2 \theta$ values of the prepared sample.

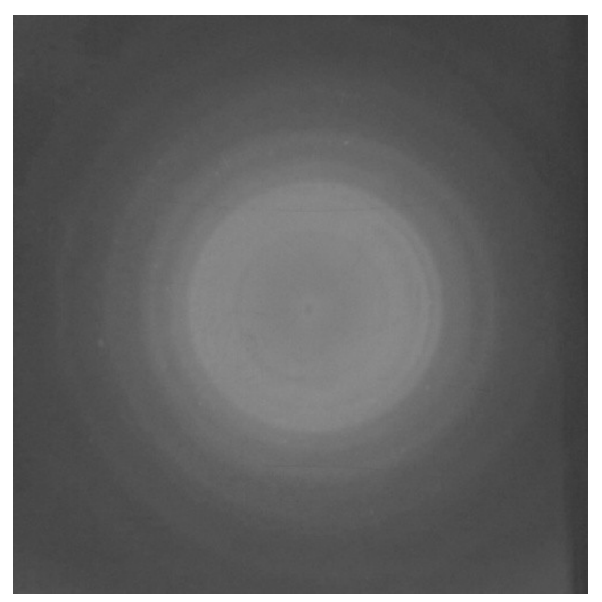

Fig.1.Hull pattern of $\mathrm{Bi}_{2} \mathrm{Te}_{3}$

Fig.2 shows the temperature dependence of Resistance (R) and Electrical conductivity $(\sigma)$ over the investigated range of 300-500 K. Current was kept constant at $2 \mathrm{~mA}$. The resistance was plotted as a function of temperature for different thicknesses is as shown in Fig.2.a). The resistance is found to have a linear dependence with the inverse of the thickness at all the temperatures. Resistivity of the samples decreases with increase in the temperature [27]. The electrical conductivity ( $\sigma)$, as shown in Fig.2.b), is found to increase with increase in the temperature, which shows semiconducting behaviour of the prepared samples. 


\section{Table 1}

\begin{tabular}{|l|l|l|l|l|l|}
\hline Sr.no. & $\begin{array}{l}\text { Diameter of the } \\
\text { rings D }(\mathrm{cm})\end{array}$ & $\begin{array}{l}\text { Radius of the } \\
\text { rings } \mathrm{r}(\mathrm{cm})\end{array}$ & $\Theta=1 / 2 \tan ^{-1}(\mathrm{r} / \mathrm{D})$ & $2 \Theta=\tan ^{-1}(\mathrm{r} / \mathrm{D})$ & $\operatorname{Sin} \theta$ \\
\hline 1 & 1.6 & 0.8 & 8.8723 & 17.7446 & 0.1542 \\
\hline 2 & 2.0 & 1.0 & 10.9007 & 21.80140 & 0.1891 \\
\hline 3 & 2.7 & 1.35 & 14.1845 & $\begin{array}{l}28.3690 \\
(100 \%)\end{array}$ & 0.2450 \\
\hline 4 & 4.0 & 2.0 & 19.3299 & 38.6598 & 0.3310 \\
\hline 5 & 4.6 & 2.3 & 21.3070 & 42.6140 & 0.3633 \\
\hline
\end{tabular}

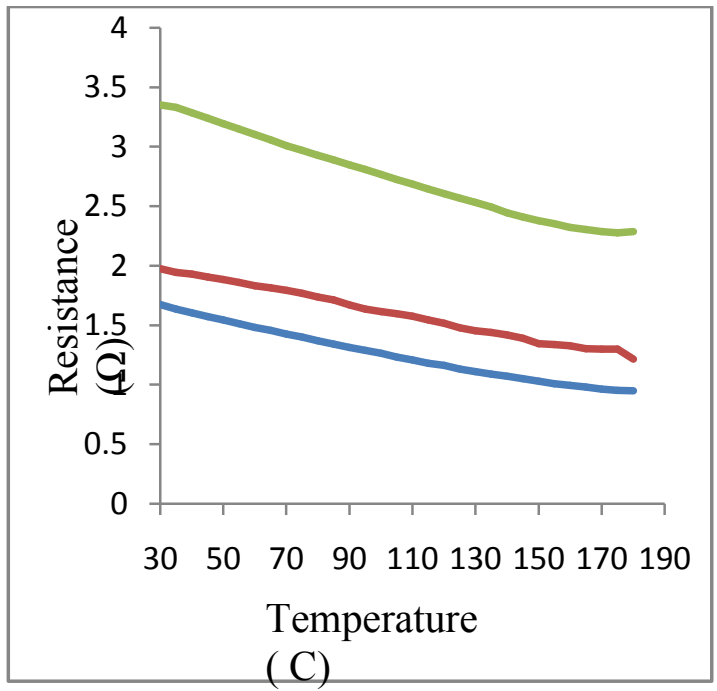

a)

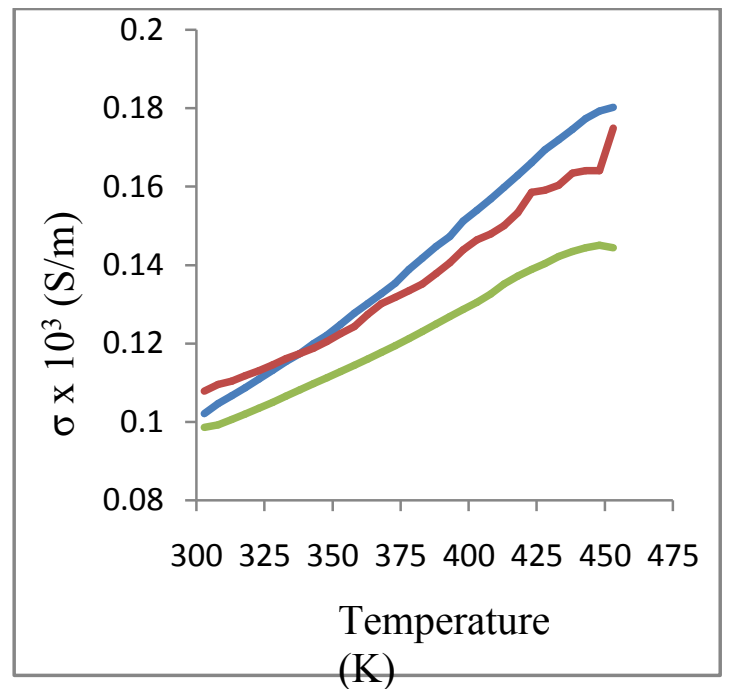

b)

Fig.2. Thermoelectric transport measurements vs. Temperature of bulk $\mathrm{Bi}_{2} \mathrm{Te}_{3}$ a) Resistance (R), b) Electrical conductivity ( $\sigma$ ).

\section{Conclusion}

In summary, we have successfully synthesised bismuth telluride $\left(\mathrm{Bi}_{2} \mathrm{Te}_{3}\right)$ by using a new developed method similar to that of a solvothermal method. The samples have shown rhombohedral phase with $\mathrm{R}^{-} \mathrm{m}$ space group. The lattice parameters values of our samples are in good agreement with those of the reported ones. It has shown a semiconducting type of behaviour with increasing temperature. 


\section{References:}

A. Boyer, and E. Cisse, (1992). Mater. Sci. Engng B 113, 103.

D.M. Rowe, and C.M. Bhandari, (1983). Modern Thermoelectrics, Holt, Rinehart and Winston, London, p. 103.

B.M. Goltsman, V.A. Kudinov, and I.A. Smirnov, (1972). Semiconducting Thermoelectric Materials Based on BiTe, Nauka, Moscow, 320.

F.J. DiSalvo, (1999). Science 285, 703.

D. M. Rowe, (1995). Handbook of Thermoelectroics, CRC Press, Boca Raton, $F L,$.

L.D. Hicks, and M.S. Dresselhaus, (1993). Phys. Rev. B 47, 12727.

L.D. Hicks, and M.S. Dresselhaus, (1993). Phys. Rev. B 47, 16631.

S.H. Yu, and M. Yoshimura, (2002). Adv. Mater. 14, 296.

Weidong Shi, Liang Zhou, Shuyan Song, Jianhui Yang, and Hongjie Zhang, (2008) . Adv. Mater. 20, 1892-1897.

Srashti Gupta, S. Neeleshwar, Vinod Kumar, Y.Y. Chen, (2012). Adv. Mat. Lett. 3(1) 50-54.

Yuzuru Miyazaki, and Tsuyoshi Kajitani, (2001). Journal of Crystal Growth 229, 542-546.

Yuan Denga, Xi-song Zhoua, Guo-dan Weia, Jing Liua, Ce-Wen Nana, and Shu-jing Zhaob, (2002). Journal of Physics and Chemistry of Solids 63, 2119-2121.

Ioffe, A.F., Semiconductor Thermoelements (Nauka, Moscow, 1956) (in Russian); or Ioffe, A.F., Semiconductor Thermoelectric and Thermoelectric Cooling (Infosearch, London, 1957).

Goldsmid, H.J.; and Douglas, R.W. (1954), Thermoelectric Br. J. Appl. Phys. $5,458$.

D.A. Wright, (1958). Nature 181, 834.

J R Drabble and R Wolfe, (1956) . Proc. Phys. Soc. B 69, 1101. 
H. J. Goldsmid, (1958). Proc. Phys. Soc. 71, 633.

Y. L. Chen, J. G. Analytis, J. H. Chu, Z. K. Liu, S. K. Mo, X. L. Qi, H. J. Zhang, D. H. Lu, X. Dai, Z. Fang, S. C. Zhang, I. R. Fisher, Z. Hussain, and Z. X. Shen, (2009). Science 325, 178.

L. Fu, (2009). Phys. Rev. Lett. 103, 266801.

Hasan, M. Z., H. Lin, and A. Bansil, (2009). Physics 2, 108.

Alpichshev, Z., J. G. Analytis, J. H. Chu, I. R. Fisher, Y. L.Chen, Z. X. Shen, A. Fang, and A. Kapitulnik, (2010). Phys. Rev. Lett. 104, 016401.

T.J. Groshens, R.W. Gedridge, and C. Lowe-Ma, (1994). Chem. Mater. 6, 727.

J.J. Ritter, and P. Maruthamuthu, (1995). Inorg. Chem. 34, 4278.

S.H. Yu, J. Yang, Y.S. Wu, Z.H. Han, J. Lu, Y. Xie, Y.T. Qian, (1998). J. Mater. Chem. 8, 1949.

H.J. Goldsmid, (1958). Proc. Phys. Soc. London 72, 17.

For a review see Qi, X.-L.; Zhang, S.-C. (2010). Physics Today, January 3338.

Bernevig, B.A.; Hughes, T.L.; and Zhang, S.-C. (2006). Science, 314, 1757; Konig, M., et al., (2007). Science, 318, 766.

Mohsin Saleeni, Muhammet S. Toprak, et.al., (2012). J. Mater. Chem. 22, 725. 